\title{
Efficacy of an aluminium triformate mouthrinse during the maintenance phase in periodontal patients: a pilot double blind randomized placebo-controlled clinical trial
}

Adriano Azaripour ${ }^{1} 2^{*}$, Jens Weusmann ${ }^{1}$, Carl Eschig ${ }^{1}$, Irene Schmidtmann ${ }^{3}$, Cornelis J. F. Van Noorden ${ }^{2}$ and Brita Willershausen ${ }^{1}$

\begin{abstract}
Background: The aim of this prospective placebo-controlled pilot study was to evaluate short-term effects of a mouthrinse containing aluminium triformate (ATF) on gingival inflammation and plaque formation in periodontal patients who are in the maintenance phase. ATF has styptic (astringent) and anti-inflammatory effects.

Methods: Forty non-smoking periodontal patients with modified sulcus bleeding index (MSBI) $\geq 40 \%$ were randomly divided into two groups. The participants received a masked mouthrinse (ATF or placebo) and were instructed with the rinsing protocol of 3 daily rinses during $30 \mathrm{~s}$ for 7 days. One blinded investigator (CE) performed all clinical examinations. The primary outcome was reduction in gingival inflammation as measured by MSBI. The secondary outcomes were reduction of the amount of plaque as measured by plaque index (PI) and approximal plaque index (API) and the occurrence of side effects. The patients were evaluated at the start and the end of the rinsing period, including the compliance of the patients.

Results: MSBI was reduced in both groups compared to baseline, but the ATF group showed significantly more reduction in MSBI compared to the placebo group (ATF: $17.6 \%$, placebo: $7.6 \%, p=0.035$ ). ATF and placebo had no effects on dental plaque. Patients reported ATF mouthrinse not to have side effects other than oral sensation, whereas compliance of the patients was good. Almost all patients in the ATF group reported reduction of gum bleeding after 1 week of rinsing with ATF.
\end{abstract}

Conclusions: This short-term pilot clinical trial is a firm basis to design a long-term controlled clinical trial to show whether ATF helps to inhibit further periodontal breakdown in maintenance patients with high MSBI.

Trial registration: This trial was registered in the WHO International Clinical Trials Registry Platform as DRKS00007672, date of registration: 21/01/2015.

Keywords: Mouthrinse, Periodontal maintenance, Gingivitis, Dental plaque

\footnotetext{
* Correspondence: adrianoasso@hotmail.com

${ }^{1}$ Department of Operative Dentistry and Periodontology, University Medical

Center, Johannes Gutenberg University Mainz, Augustusplatz 2, 55131 Mainz,

Germany

2Department of Cell Biology and Histology, Academic Medical Center,

University of Amsterdam, Amsterdam, The Netherlands

Full list of author information is available at the end of the article
}

(c) 2016 Azaripour et al. Open Access This article is distributed under the terms of the Creative Commons Attribution 4.0 International License (http://creativecommons.org/licenses/by/4.0/, which permits unrestricted use, distribution, and reproduction in any medium, provided you give appropriate credit to the original author(s) and the source, provide a link to the Creative Commons license, and indicate if changes were made. The Creative Commons Public Domain Dedication waiver (http://creativecommons.org/publicdomain/zero/1.0/) applies to the data made available in this article, unless otherwise stated. 


\section{Background}

The link between oral health and general health is becoming generally accepted. Prevention of oral inflammatory diseases is considered to be a key element of good oral and systemic health [1]. Tools and chemical compounds in addition to mechanical plaque control may be helpful to support oral health. Additional chemical plaque control as part of domestic oral hygiene has always been playing an important role in the treatment of gingival inflammation $[2,3]$.

Patients with periodontitis need to be enrolled in a periodontal treatment protocol. During the initial visit, the patient's medical and dental history is evaluated and clinical examinations and radiographic analyses are performed. The patients are being informed that periodontitis is an irreversible disease and that progress can be arrested by proper treatment. Periodontal treatment includes non-surgical and surgical procedures and domestic oral hygiene regimes. The maintenance phase after periodontal treatment is as important as the treatment itself and plays a decisive role whether the long-term outcome is successful or not [4]. Continuous domestic oral hygiene is indispensable to reach this aim and suitable antiseptics may help.

Chlorhexidine digluconate (CHX) is currently considered to be the most effective antiseptic mouthrinse due to its high substantivity and strong anti-bacterial effects and has been used in dentistry for many decades as liquid or gel [5]. Numerous studies have confirmed its efficacy in inhibition of plaque formation and reduction of gingival inflammation $[6,7]$. CHX-containing mouthrinses have a significant antibacterial effect up to $7 \mathrm{~h}$ after its application [8]. Its efficacy is similar in mouthrinses with and without alcohol [9]. However, CHX has side effects such as reversible dysgeusia, black hairy tongue and tooth discolorations $[5,10-12]$ and is therefore not suitable for daily use in long-term periodontal maintenance. As a consequence, the search for effective alternatives without side effects continues.

Novel rinsing solutions should be tested for their application in periodontal maintenance for efficacy and side effects. Aluminium triformate (ATF), an aluminium salt, has been used for several decades in Europe to control "bleeding gums" and is considered to be safe for daily use. Other compounds of aluminium salts (aluminium-containing mouthrinses) have shown a reducing effect on bacterial growth and plaque formation [13, 14]. Application of alum (in combination with salt and vinegar) for mouth rinsing was advocated by Hippocrates approx. 2400 years ago [15]. Aluminium and other similar metals (polyvalent cations) are widely used in dental products [14]. ATF is available since 1967, but besides some clinical trials in the 80ies of last century that did not meet todays' standards of good clinical practice, studies have not been performed so far to test its efficacy in the periodontal maintenance phase. ATF is likely anti-inflammatory due to its astringent characteristics, but data are not available for its effects on gingival inflammation and plaque formation. ATF creates a protective surface of denaturated keratins and other proteins as a colloidal layer through ionic bonds with proteins of the gingiva preventing penetration of bacterial compounds such as endotoxins into the underlying connective tissue.

The aim of this clinical short-term pilot study was to analyze the effects of ATF as active component in a commercially-available mouthrinse (Cional ${ }^{\circ}$; Kreussler \& Co. GmbH, Wiesbaden, Germany) as an adjunct to mechanical oral hygiene in the maintenance phase of periodontal patients. The primary hypothesis was that the use of ATF leads to a stronger reduction of gingival inflammation than placebo. The secondary hypothesis was that the use of ATF reduces plaque formation and does not have significant side effects whereas the compliance of patients is good.

\section{Methods}

\section{Experimental design}

A pilot randomized placebo-controlled, 1-week double blind clinical trial with parallel groups was designed. The mouthrinse containing ATF is freely available without prescription at the pharmacy to be used for a period of maximally one week. We considered it necessary first to perform a short-term study as a pilot for an extensive clinical trial for two reasons: $1^{\circ}$ we wanted to test the compliance of the patients who participated in the study and $2^{\circ}$ we wanted to monitor side effects. Side effects such as allergic reaction or skin irritation have been reported as possible side effects. However, ATF-containing mouthrinse has been used for over 30 years and side effects have not been reported thus far.

\section{Study participants}

Consecutive periodontal patients were screened at the Department of Operative Dentistry at the University Medical Center (Mainz, Germany) during August and September 2013. Patients who met the inclusion and exclusion criteria were recruited for enrollment.

The following patient inclusion criteria were applied:

- History of chronic periodontitis, having had active periodontal treatment including non-surgical and surgical therapies, and being in maintenance for $\geq 1$ year;

- $\geq 18$ years old;

- Willing to participate and willing to sign the informed consent;

- Having a modified sulcus bleeding index (MSBI) of $\geq 40 \%$ [16];

- No pocket depths $\geq 6 \mathrm{~mm}$;

- At least 20 teeth (at least 5 teeth in each quadrant);

- Systemically healthy. 
Exclusion criteria:

- Known hypersensitivity to ATF;

- Known hypersensitivity to other ingredients of Cional $^{\circ}$ (Cremophor RH40, peppermint oil, mint oil, propylene glycol, glycerol);

- Any anti-inflammatory or antibiotic therapy $<3$ months before treatment;

- Any other treatment during the study;

- Smoker;

- Pregnancy or wish for pregnancy;

- Lactating women;

- Participation in another clinical study;

- Lack of ability to participate in the screening appointments.

\section{Interventions}

\section{Screening visit}

After a careful medical health evaluation, the oral health examination was carried out at the screening visit. The investigator (CE) controlled the inclusion/exclusion criteria and informed the patients about the details and aim of the study. The subjects signed the informed consent form when they accepted to participate voluntarily in the clinical trial.

\section{Baseline visit}

At the baseline visit, each participant was orally investigated (see below) and received an individual trial number. Following the recruitment, the patients were randomly allocated to either test or control group. Randomization was performed using random numbers from a computer-generated list provided by the manufacturer. Both, dentists and participants were masked to group allocation. All patients received their assigned product kit containing a non-labeled bottle (coded with numbers) of a concentrate that contained either a solution of ATF or the same solution but without ATF (placebo) and a special scaled container to dilute the rinsing solution with water. The patients were trained to mix the solution and each subject was given a written instruction (rinsing protocol) on how to use and dilute the mouthrinse. The rinsing solution consisted of $2.5 \mathrm{ml}$ ATF or placebo from the masked bottle, which was to be diluted with water to $50 \mathrm{ml}$ (Fig. 1). The participants were instructed to rinse 3 times daily for $30 \mathrm{~s}$.

To check the compliance, the subjects had to make a note of the date, the time, the solution volume in milliliters and the rinsing time in seconds. The rinsing protocol served both to control the duration of the rinse and the motivation of the patient in terms of a memory aid. Furthermore, the subjects were asked to complete a questionnaire concerning the taste of the product, special sensation on tongue or mucosa and possible loss of taste sensation.

\section{One-week reevaluation}

After one week of rinsing, the used bottles as well as compliance forms were collected and the patients were orally investigated (see below) and asked for occurrence of any adverse effects.

\section{Clinical parameters}

One calibrated examiner (CE), who was blinded for the content of the mouthrinse, performed all oral investigations. The gingival condition was evaluated during the baseline visit using MSBI (Additional file 1) and plaque was measured using the plaque index (PI) [17] and approximal plaque index (API) (Additional file 1) using plaque disclosing tablets (PD Produits Dentaires SA, Vevey, Switzerland) for $30 \mathrm{~s}$ and subsequent water rinsing. The clinical examinations were repeated after one week of rinsing with ATF or placebo mouthrinse (follow-up visit).

\section{Statistical analysis}

As the current trial is a pilot study, no formal calculation on power and group sizes could be made a priori. Categorical parameters were described using absolute and relative frequencies, continuous parameters were

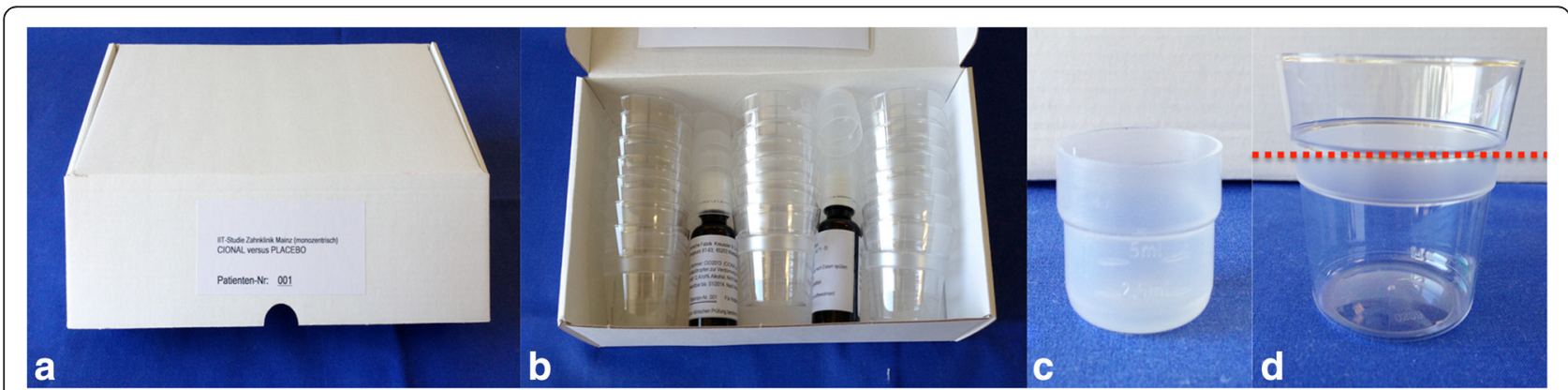

Fig. 1 Preparation of the rinsing solution. Each participant received a box containing two bottles of the rinsing concentrate (a and $\mathbf{b})$. Special cups of $2.5 \mathrm{ml}(\mathbf{c})$ and $50 \mathrm{ml}(\mathbf{d})$ were included in the box for the preparation of the rinsing solution. The special cup of $2.5 \mathrm{ml}$ (c) was used to measure the mouth rinse concentrate that has to be diluted with water in the cup of $50 \mathrm{ml}(\mathbf{d})$ 
described by mean, standard deviation and range. The age of the patients in the test and control group was analyzed for differences by the Student t-test. The gender distribution was analyzed by the Fisher's exact test.

The primary endpoint of this study was MSBI. The baseline and MSBI follow-up levels were compared between groups using the Mann-Whitney test. The Wilcoxon signed-rank test within each group (baseline and follow-up visit) was used to assess the change in MSBI for ATF and placebo treatment. PI and API were analyzed in the same way.

Statistical analyses were performed using SPSS 22.0 (Chicago, IL, USA) and SAS 9.4 (Cary, NC, USA, 2002-2012) and the significance level was set at $\alpha=$ 0.05. Sample size calculation was performed using nQuery Advisor 5.0 (1995-2002, Janet Elashoff).

\section{Results}

\section{Study population and patient characteristics}

A total of 101 periodontal patients were assessed for eligibility and 42 met the inclusion and exclusion criteria and were enrolled (Fig. 2). One patient declined to participate and one patient withdrew. Thus, 40 patients were randomized and equally allocated to either the ATF group or the placebo group. All participants $(n=$ 40) continued until the end of study and for all patients complete data sets were available (Fig. 2).

A total of 101 periodontal patients were assessed for eligibility. The age of participants ranged between 31 and 78 years and the majority of patients was female (65\% in the ATF group and $90 \%$ in the placebo group). The mean age (60.6 and 58.6, respectively) did not differ between the ATF group and placebo group (Table 1). The number of teeth per group was comparable (25.9 teeth in the ATF group and 24.4 in the placebo group). The relative frequency of residual pockets was similar as well in the ATF group and placebo group $(18.1 \pm 12.3$ and $17.1 \pm 13.1 \%$, respectively; Table 1 ).

\section{Clinical outcomes}

The analysis of the data showed a notable improvement of the MSBI values in both groups after one week of rinsing (a reduction from 62.8 to $45.2 \%$ for the ATF group and from 59.2 to $51.6 \%$ for the placebo group at baseline and after 1 week rinsing, respectively; Table 2). The reduction in MSBI was significantly higher in the $\operatorname{ATF}$ group $(p=0.035)$.

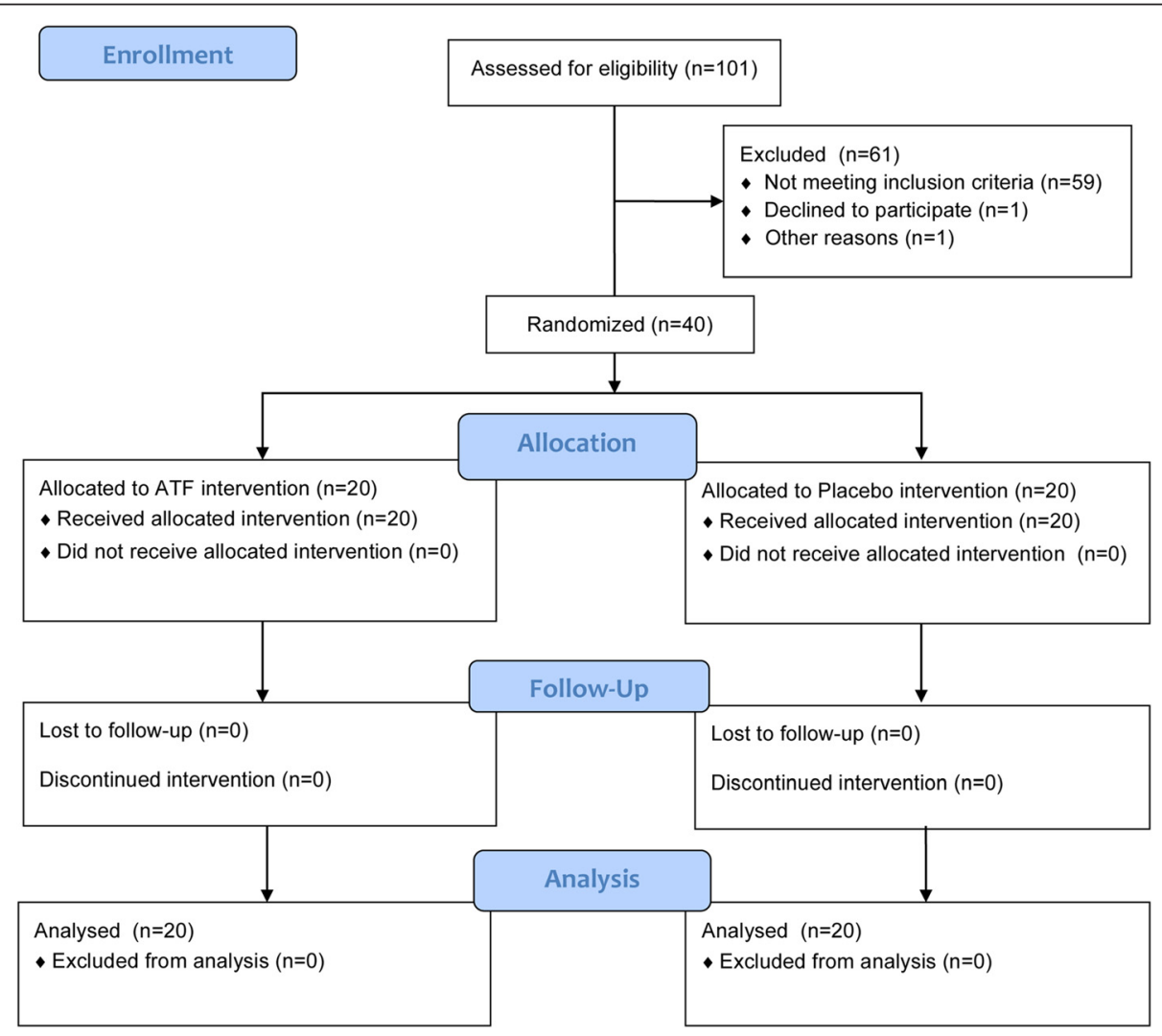

Fig. 2 Study flow diagram according to CONSORT 2010 
Table 1 Demographic data of the patients included in the study

\begin{tabular}{llll}
\hline Group & $\operatorname{ATF}(n=20)$ & Placebo $(n=20)$ & $p$-Value \\
\hline Age & $60.6 \pm 9.4$ & $58.6 \pm 10.1$ & $31-75$ \\
$\quad$ Range & $46-78$ & & 0.636 \\
Gender & & $18(90 \%)$ & 0.058 \\
$\quad$ Female & $13(65 \%)$ & $2(10 \%)$ & $24.3 \pm 2.5$ \\
$\quad$ Male & $7(35 \%)$ & $146.7 \pm 15.7$ \\
Number of teeth & $25.8 \pm 2.9$ & $129-174$ & 0.445 \\
Number of sites & $155.4 \pm 18.5$ & $26.7 \pm 21.1$ & 0.149 \\
$\quad$ Range & $120-186$ & $(9-93)$ & $17.1 \pm 13.1$ \\
Number of residual pockets (4-5 mm) & $28 \pm 20.3$ & 0.440 \\
$\quad$ Range & $(1-86)$ & $18.1 \pm 12.3$ & 0.398 \\
\hline Frequency of residual pockets (\%)
\end{tabular}

Values represent descriptive means and standard deviations

Oral hygiene and the amount of dental plaque were evaluated, analyzing PI and API. The differences of PI and API at baseline and after 1 week of rinsing were not significant $(p=0.947$ for PI and $p=0.495$ for API; Table 2$)$.

\section{Compliance and subjective outcomes}

All subjects $(n=40)$ returned the forms containing details about date, solution volume and rinsing time. All patients had rinsed during the entire week. Table 3 shows the subjective outcomes of the participants with respect to taste, oral sensations and personal assessment during the rinsing time. None of the participants complained about an unpleasant taste or negative or adverse effects (Table 3).

On the other hand, patients did report on oral sensation. The majority of participants that had applied the ATF mouthrinse reported mouth dryness directly after rinsing, roughness of the surface of the teeth, tongue and mucosa, astringent feeling of the mucosa, furry sensation of the oral cavity after rinsing and metallic taste shortly after rinsing. Interestingly, 19 out of 20 participants in the ATF group reported a reduction of gum bleeding during domestic oral hygiene procedures (Table 3).

\section{Discussion}

The present double blind placebo-controlled pilot trial is the first in vivo study that evaluates the efficacy of an ATF-containing mouthrinse used by periodontal patients in the maintenance phase. Fourty patients were enrolled and have completed this study. The primary hypothesis of this study was that ATF reduces the MSBI more than placebo without significant side effects has been confirmed.

Table 2 Clinical parameters at baseline and after one week rinsing (reevaluation)

\begin{tabular}{|c|c|c|c|c|}
\hline \multicolumn{2}{|c|}{ Clinical parameter } & $\operatorname{ATF}(n=20)$ & Placebo $(n=20)$ & $p$-value (inter-group differences) \\
\hline \multicolumn{5}{|c|}{ Primary outcome: } \\
\hline \multirow[t]{4}{*}{ MSBI } & Baseline & $62.8 \pm 17.1$ & $59.2 \pm 14.8$ & 0.529 \\
\hline & Reevaluation & $45.2 \pm 18.9$ & $51.6 \pm 19.5$ & 0.383 \\
\hline & $p$-value (intra-group differences) & 0.001 & 0.018 & \\
\hline & Reduction & $17.6 \pm 13.8$ & $7.6 \pm 11.3$ & 0.035 \\
\hline \multicolumn{5}{|c|}{ Secondary outcome: } \\
\hline \multirow[t]{4}{*}{ PI } & Baseline & $0.7 \pm 0.5$ & $0.7 \pm 0.4$ & 0.640 \\
\hline & Reevaluation & $0.8 \pm 0.5$ & $0.7 \pm 0.4$ & 0.445 \\
\hline & $p$-value (intra-group differences) & 0.17 & 0.34 & \\
\hline & Reduction & $0.09 \pm 0.27$ & $0.06 \pm 0.26$ & 0.947 \\
\hline \multirow[t]{4}{*}{ API } & Baseline & $52.2 \pm 19.1$ & $43.3 \pm 14.7$ & 0.157 \\
\hline & Reevaluation & $57.3 \pm 20.8$ & $45.1 \pm 15.9$ & 0.056 \\
\hline & $p$-value (intra-group differences) & 0.177 & 0.602 & \\
\hline & Reduction & $5.1 \pm 14.9$ & $1.8 \pm 12.0$ & 0.495 \\
\hline
\end{tabular}


Table 3 Subjective outcomes

\begin{tabular}{|c|c|c|}
\hline & $\begin{array}{l}\text { ATF } \\
(n=20)\end{array}$ & $\begin{array}{l}\text { Placebo } \\
(n=20)\end{array}$ \\
\hline \multicolumn{3}{|l|}{ Taste } \\
\hline Unpleasant & 0 & 0 \\
\hline Pleasant & 0 & 20 \\
\hline Reduction in taste sensation & 0 & 0 \\
\hline \multicolumn{3}{|l|}{ Oral sensation } \\
\hline Mouth dryness directly after rinsing & 19 & 0 \\
\hline $\begin{array}{l}\text { Roughness surface of the teeth, tongue and } \\
\text { mucosa }\end{array}$ & 20 & 0 \\
\hline Astringent feeling of the mucosa & 18 & 0 \\
\hline Furry sensation of the oral cavity after rinsing & 19 & 0 \\
\hline Metallic taste shortly after rinsing & 19 & 0 \\
\hline $\begin{array}{l}\text { Reduction of the gum bleeding during domestic } \\
\text { oral hygiene procedures }\end{array}$ & 19 & 6 \\
\hline
\end{tabular}

Values represent absolute numbers of individuals with positive responses

After a period of 7 days, there was a notable reduction in gingival bleeding in both groups. The comparison between the two groups showed a significantly higher reduction in gingival bleeding as assessed by MSBI in the ATF group. This is consistent with patients' subjective experience in the ATF group of reduced sulcus bleeding after rinsing. PI and API were not affected during this short-term application of an ATF-containing mouthrinse. It should be noted that the current rinsing period is relatively short, long-term effects of the rinsing with ATF need to be investigated. This pilot study was performed to demonstrate whether MSBI, PI and API were affected by an ATF-containing mouthrinse without side effects and whether the compliance of the patients included in the study was good.

This study was a pilot study and therefore not necessarily powered to detect significant differences between treatments. In this study, the estimated probability that ATF leads to a stronger decrease in MSBI $\left(\mathrm{P}\left(\triangle \mathrm{MSBI}_{\mathrm{ATF}}\right.\right.$ $\left.<\Delta \mathrm{MSBI}_{\text {Placebo }}\right)$ ) was 0.3125 . When calculating sample size and power under the assumption that $\mathrm{P}\left(\triangle \mathrm{MSBI}_{\mathrm{ATF}}\right.$ $\left.<\Delta \mathrm{MSBI}_{\text {Placebo }}\right)=0.3125$ and significance level $\alpha=0.05,38$ patients per group are needed to demonstrate this effect with $80 \%$ power and 50 patients are needed to demonstrate this effect with $90 \%$ power. With 20 patients per group, the calculated power is $53 \%$. Hence, further studies should include at least 100 patients in order to demonstrate efficacy of ATF.

The improvement of the MSBI values in the placebo group can be explained by the Hawthorne effect [18]. This phenomenon is considered to be based on increased attention, e.g. due to the participation in a clinical trial and the consciousness of being evaluated, leading to an improvement of productivity, or, in this case, a reduction in MSBI in the placebo group because of an improved oral hygiene during the study. However, a similar Hawthorne effect must be present in the ATF group, too. Because both patients and investigator were masked with respect to treatment, it is unlikely, that this effect differs between groups. The reduction in MSBI was significantly stronger in the ATF group than in the placebo group, which points at a true effect of ATF on MSBI.

Lang et al. [19] demonstrated the correlation between the amount of gingival bleeding and tooth survival in patients during periodontal maintenance. It was shown that periodontal patients with bleeding $\geq 16 \%$ on probing had a higher risk of losing attachment than patients with $<16 \%$ bleeding on probing. After 4 consecutive maintenance appointments, patients with no bleeding on probing had a 20 -fold lower risk of attachment loss than patients with bleeding on probing at the 4 maintenance appointments. These data indicate the importance of oral hygiene and control of gingival bleeding during the maintenance phase.

Ramberg et al. [20] showed a correlation between gingival inflammation and de novo plaque formation. Reduced gingival inflammation lowers the amount of crevicular fluid that contains proteins, which are metabolized by plaque microorganisms. As the quantity of crevicular fluid correlates with the grade of gingiva inflammation, a reduction of sulcus irritation decreases the inflammation and therefore prevents de novo plaque formation. Furthermore, Van der Velden [21] stated that gingival fluid is the major cause of de novo plaque formation. However, neither ATF rinse nor placebo rinse had any effect on the amount of dental plaque during the short experimental period of 7 days in our study. Putt et al. [14] showed a significant reduction in the amount of plaque after 2 and 4 weeks of rinsing and thus, it may well be that longer rinsing periods show effects on plaque levels as well.

With respect to the patients' evaluation reports, ATF mouthrinse seems to be safe and not causing harmful side effects. Almost all patients in the ATF group reported the rough sensation of gingiva and teeth surface accompanied with a metallic taste. These sensations can be clearly explained by the astringent properties of aluminium solutions [22]. However, these sensations disappeared after a few minutes and not one patient indicated a desire to stop the rinsing, thus it is concluded that compliance of the patients included in the study is good.

The fast reduction in gingival bleeding may be useful as a pre-surgical measure in patients with inflamed gingiva. For protocols in which authors propose immediate periodontal surgery (without initial therapy) in patients with advanced periodontitis [23], it can be recommended that patients rinse with ATF during one week before surgery; the styptic effect of ATF may well be beneficial, but this application in untreated periodontitis 
patients is not yet tested and needs further investigation. ATF rinse may also be beneficial for patients with fixed orthodontic appliances during an acute period of gingival inflammation that are at risk for temporary periodontal destructive processes [24].

\section{Conclusions}

ATF mouthrinse solution appears to be a promising adjunct to mechanical tools in the periodontal maintenance phase in our short-term pilot clinical trial. A longterm trial with a larger number of participants is needed to confirm our findings. The current study shows that the ATF rinse solution is well accepted by patients and does not have major and long-lasting side effects.

\section{Additional file}

Additional file 1: Modified SBI and API (Lange, [16]). (DOCX 73 kb)

\section{Abbreviations}

ATF: aluminium triformate; MSBI: modified sulcus bleeding index; API: approximal plaque index; Pl: plaque index.

\section{Acknowledgement}

The authors thank Mrs. N. Kary for her help in organization of this study.

\section{Source of funding statement}

The study was supported by Kreussler Pharma (Wiesbaden, Germany) through a grant to the Research Group of Department of Operative Dentistry. The study was conducted and analyzed by the authors.

\section{Availability of data and materials}

All data of individual patients are achieved in Department of Operative Dentistry and Periodontology, University Medical Center, Johannes Gutenberg University, Mainz, Germany. For data security reasons and anonymity of the patients, these files can not be shared.

\section{Authors' contributions}

AA, CE and JW carried out the study. CE performed all clinical examinations. Statistical analysis was performed by AA and IS. AA, CVN and BW conceived of the study and participated in its design and coordination. All authors read and approved the final manuscript.

\section{Approval}

The study was approved by the medical ethics committee of the Johannes Gutenberg University Medical Center (387.538.12 (8639 F)). Furthermore, the trial was registered in the WHO International Clinical Trials Registry Platform as DRKS00007672.

\section{Competing interests}

The authors declare that they have no competing interests.

\section{Consent for publication}

All participants signed the informed consent form when they accepted to participate voluntarily in the clinical trial. All patients agreed that their data would be published anonymously.

\section{Author details}

${ }^{1}$ Department of Operative Dentistry and Periodontology, University Medical Center, Johannes Gutenberg University Mainz, Augustusplatz 2, 55131 Mainz, Germany. ${ }^{2}$ Department of Cell Biology and Histology, Academic Medical Center, University of Amsterdam, Amsterdam, The Netherlands. ${ }^{3}$ Institute of Medical Biostatistics, Epidemiology and Informatics (IMBEI), Johannes Gutenberg University, Mainz, Germany.
Received: 8 December 2015 Accepted: 12 May 2016

Published online: 23 May 2016

\section{References}

1. Oral health: prevention is key. Lancet 2009 373:1. doi: 10.1016/s01406736(08)61933-9.

2. Löe $H$, Schiott CR. The effect of mouthrinses and topical application of chlorhexidine on the development of dental plaque and gingivitis in man. J Periodontal Res. 1970;5:79-83.

3. Paraskevas S, Van der Weijden GA. A review of the effects of stannous fluoride on gingivitis. J Clin Periodontol. 2006;33:1-13. doi:10.1111/j.1600051X.2005.00860.X

4. Axelsson P, Lindhe J, Nystrom B. On the prevention of caries and periodontal disease. Results of a 15-year longitudinal study in adults. J Clin Periodontol. 1991;18:182-9.

5. Van Strydonck DA, Slot DE, Van der Velden U, Van der Weijden F. Effect of a chlorhexidine mouthrinse on plaque, gingival inflammation and staining in gingivitis patients: a systematic review. J Clin Periodontol. 2012;39:1042-55. doi:10.1111/j.1600-051X.2012.01883.x.

6. Albandar JM, Gjermo P, Preus HR. Chlorhexidine use after two decades of over-the-counter availability. J Periodontol. 1994;65:109-12. doi:10.1902/jop. 1994.65.2.109.

7. Renton-Harper P, Addy M, Moran J, Doherty FM, Newcombe RG. A comparison of chlorhexidine, cetylpyridinium chloride, triclosan, and C31G mouthrinse products for plaque inhibition. J Periodontol. 1996;67:486-9. doi:10.1902/jop.1996.67.5.486.

8. Cousido MC, Tomas Carmona I, Garcia-Caballero L, Limeres J, Alvarez M, Diz P. In vivo substantivity of $0.12 \%$ and $0.2 \%$ chlorhexidine mouthrinses on salivary bacteria. Clinical Oral Investig. 2010;14:397-402. doi:10.1007/s00784-009-0320-2.

9. Leyes Borrajo JL, Garcia VL, Lopez CG, Rodriguez-Nunez I, Garcia FM, Gallas TM. Efficacy of chlorhexidine mouthrinses with and without alcohol: a clinical study. J Periodontol. 2002;73:317-21. doi:10.1902/jop.2002.73.3.317.

10. Eley BM. Antibacterial agents in the control of supragingival plaque-a review. Br Dent J. 1999;186:286-96.

11. Gent JF, Frank ME, Hettinger TP. Taste confusions following chlorhexidine treatment. Chem Senses. 2002;27:73-80.

12. Gurgan CA, Zaim E, Bakirsoy I, Soykan E. Short-term side effects of $0.2 \%$ alcohol-free chlorhexidine mouthrinse used as an adjunct to non-surgical periodontal treatment: a double-blind clinical study. J Periodontol. 2006;77: 370-84. doi:10.1902/jop.2006.050141.

13. Simonsson T, Glantz PO, Edwardsson S. Effects of cations on the colloidal stability of some oral bacteria. Acta Odontol Scand. 1988;46:83-7.

14. Putt MS, Kleber CJ, Smith CE. Evaluation of an alum-containing mouthrinse in children for plaque and gingivitis inhibition during 4 weeks of supervised use. Pediatr Dent. 1996;18:139-44.

15. Mandel ID. Chemotherapeutic agents for controlling plaque and gingivitis. J Clin Periodontol. 1988:15:488-98.

16. Lange DE. Parodontologie in der täglichen Praxis. Berlin: QuintessenzVerlag; 1986.

17. Silness J, Löe H. Periodontal disease in pregnancy. II. Correlation between oral hygiene and periodontal condition. Acta Odontol Scand. 1964:22:121-35.

18. Parsons HM. Hawthorne: an early OBM experiment. J Organ Behav Manag. 1991:12:27-43.

19. Lang NP, Joss A, Tonetti MS. Monitoring disease during supportive periodontal treatment by bleeding on probing. Periodontol. 1996;12:44-8

20. Ramberg $P$, Lindhe J, Dahlen G, Volpe AR. The influence of gingival inflammation on de novo plaque formation. J Clin Periodontol. 1994:21:51-6.

21. Van der Velden $U$. The significance of supragingival plaque accumulation in periodontal disease. Int J Dent Hyg. 2006;4 Suppl 1:11-4. doi:10.1111/j.16015037.2006.00196.x. discussion 50-2.

22. Mourughan K, Suryakanth MP. Evaluation of an alum-containing mouthrinse for inhibition of salivary streptococcus mutans levels in children-a controlled clinical trial. J Indian Soc Pedod Prev Dent. 2004;22:100-5.

23. Miremadi SR, De Bruyn H, Steyaert H, Princen K, Sabzevar MM, Cosyn J. A randomized controlled trial on immediate surgery versus root planing in 
patients with advanced periodontal disease: a cost-effectiveness analysis. J Clin Periodontol. 2014;41:164-71. doi:10.1111/jcpe.12201.

24. Azaripour A, Weusmann J, Mahmoodi B, Peppas D, Gerhold-Ay A, Van Noorden CJ, Willershausen B. Braces versus Invisalign ${ }^{\oplus}$ : gingival parameters and patients' satisfaction during treatment: a cross-sectional study. BMC Oral Health. 2015;15:69. doi:10.1186/s12903-015-0060-4.

Submit your next manuscript to BioMed Central and we will help you at every step:

- We accept pre-submission inquiries

- Our selector tool helps you to find the most relevant journal

- We provide round the clock customer support

- Convenient online submission

- Thorough peer review

- Inclusion in PubMed and all major indexing services

- Maximum visibility for your research

Submit your manuscript at www.biomedcentral.com/submit 